Boletín de la Sociedad Zoológica del Uruguay, 2021

Vol. 30 (1): 1-13

ISSN 2393-6940

https://journal.szu.org.uy

DOI: https://doi.org/10.26462/30.1.1

\title{
¿CUÁNTO CONOCEMOS DE NUESTRA FAUNA AUTÓCTONA? UN ESTUDIO SOBRE VERTEBRADOS TETRÁPODOS
}

\author{
Martín Buschiazzo \\ Museo de Historia Natural Dr. Carlos A. Torres de la Llosa. DGES. \\ Autor para correspondencia: buschiazzomartin@gmail.com
}

Fecha de recepción: 22 de marzo de 2021

Fecha de aceptación: 25 de mayo de 2021

\section{RESUMEN}

Desde los comienzos de la civilización y en diferentes culturas las personas han establecido una fuerte conexión con los animales y en muchas tradiciones culturales les ha inculcado de un valor simbólico. Un buen conocimiento de las especies de fauna y flora de un lugar determinado, permite llegar a comprender la importancia de su protección y conservación, lo que en gran medida ayudará frente a uno de los principales problemas de la humanidad a escala global: la pérdida de biodiversidad. En los últimos años en Uruguay ha existido un aumento respecto al conocimiento de las especies que integran nuestra fauna autóctona, si bien esta información es reconocida y accesible dentro del ámbito científicoacadémico, muchas veces no llega a conocerse por el resto de la población. El objetivo del presente trabajo es evaluar el estado actual del conocimiento de la fauna de vertebrados tetrápodos autóctonos en la población uruguaya a través de una encuesta semi-estructurada a 593 personas. Los resultados obtenidos mostraron un conocimiento escaso de nuestra fauna, hecho que se ha visto en otros países. Se discute el desafío de implementar acciones y estrategias que favorezcan el conocimiento de la misma en la población.

Palabras Clave: Fauna autóctona, Conocimiento de fauna, Conservación, Uruguay.

\footnotetext{
ABSTRACT

How much do we know about our native fauna? A study on tetrapod vertebrates. From the beginning of civilization and in several cultures, people established a strong connection with animals and in many cultural traditions they have imbued them with a symbolic value. A good knowledge of the species of fauna and flora of a certain place, allows us to understand the importance of their protection and conservation, which will greatly help to face one of the main problems of humanity on a global
}

scale: the loss of biodiversity. In recent years in Uruguay there has been an increase regarding the knowledge of the species that make up our native fauna. Although this information is recognized and accessible within the scientific-academic field, it is often not known by the rest of the population. The objective of this work is to evaluate the current state of knowledge of the fauna of native tetrapod vertebrates in the Uruguayan population, which was done through a semi-structured survey of 593 people. The results obtained showed a scarce knowledge of our fauna, a fact that has been seen in other countries. We discuss the challenge of implementing actions and strategies that favor the knowledge about it in the population.

Keywords: Native fauna, Fauna knowledge, Conservation, Uruguay

\section{INTRODUCCIÓN}

Los seres humanos hemos estado vinculados al resto de los animales durante los procesos evolutivos, utilizando a la fauna como promotora de nuestro bienestar (Almeida, García Fernández y Stretch-Ribeiro, 2019). Desde los comienzos de la civilización, la fauna ha constituido un recurso importante para las poblaciones humanas (Rodas, Estrada, Rau y Morales, 2016), donde su aprovechamiento y valoración han estado ligado a aquellas que representan recursos alimenticios, económicos, medicinales, religiosos o de otra índole; formando parte de la cultura (Barrera y Toledo, 2005; Velarde, 2012). Pero esta relación instrumental es solo una parte de la conexión del ser humano con la fauna en general. A su vez también se ha desarrollado la curiosidad sobre los seres vivos y la propensión a generar empatía e incluso miedo a los animales (Almeida et al., 2019). De acuerdo con la hipótesis de la "Biofilia" propuesta por Wilson (1984) 
los seres humanos a través del contacto durante miles de años han desarrollado la tendencia innata de afiliarse con otros seres vivos y sus procesos. De hecho, a través del tiempo y en diferentes culturas las personas han establecido una fuerte conexión con los animales y en muchas tradiciones culturales se les ha inculcado de un valor simbólico. (Walsh, 2009).

En las últimas tres décadas nuevos paradigmas han recibido la atención de la humanidad, entre éstos: podemos destacar el uso sustentable de los bienes comunes naturales y la conservación de la diversidad biológica (Kremen, Merenlender y Murphy, 1994). Un buen conocimiento de las especies de fauna y flora de un lugar determinado, permite llegar a comprender la importancia de su protección y conservación, lo que en gran medida ayudará frente a uno de los principales problemas de la humanidad a escala global: la pérdida de biodiversidad (Arias Rodríguez, Torralba-Burrial, Anadón Álvarez, Lastra López, García y Herrero Vázquez, 2018). En efecto, el Convenio sobre Diversidad Biológica (CDB) de 1992 afirma que la conservación de la diversidad biológica es un interés común de toda la humanidad (Glowka, BurhenneGuilmin y Synge, 1994) y la necesidad de actuar para mitigar su pérdida se encuentra recogida en varios de los Objetivos de Desarrollo Sostenible (ODS) de la Organización de las Naciones Unidas. Asimismo el conocimiento de las características y hábitats de los animales es esencial para entender sus características zoológicas, ecológicas y ambientales (Patrick y Tunnicliffe, 2011). Así, el éxito en la conservación de la fauna silvestre, no sólo depende de aspectos biológicos, sino también de cuestiones sociales y culturales por parte de las sociedades humanas; esto es lo que Bath (1996) denominó "la dimensión humana en el manejo de recursos". Paradójicamente, en los últimos años se ha estado generando (y aún sigue produciéndose) una desconexión cada vez mayor entre la sociedad y el conocimiento de la naturaleza, con incidencia tanto en la población adulta (Melero-Alcíbar y Gamarra, 2016) como infantil (Ballouard, Brischoux y Bonnet, 2011; Genovart, Tavecchia, Enseñat y Laiolo, 2013). En la actualidad asistimos a un continuo e intenso deterioro de los saberes populares. La civilización industrial tiene la conciencia de haberse aislado de la Naturaleza (Clavero y De Los Reyes, 2003). La fe en las soluciones tecnológicas del "progreso" ha acabado con la mayor parte de los conocimientos que sirven de sustento a los sistemas tradicionales de explotación de la Naturaleza (Clavero y De Los Reyes, 2003). La falta de conocimientos de las personas en cuanto a la vida silvestre nos ha llevado a la pérdida de la biodiversidad (Narvaez, Cueva y Maldonado, 2019). De hecho, se ha propuesto como una de las medidas para avanzar en la transición hacia la "sustentabilidad", un incremento en la educación sobre la naturaleza en la infancia, lo que generaría a mediano plazo una mayor concienciación en el conjunto de la sociedad (Ripple et al., 2017).

En los últimos años, en Uruguay ha existido un aumento respecto al conocimiento de las especies que integran nuestra fauna autóctona. En 2013 se reconocían 117 especies de mamíferos, 455 de aves, 71 de reptiles y 48 de anfibios (Soutullo, Clavijo y Martínez-Lanfranco, 2013), aunque dicho número continúa en aumento sobre la base de nuevos descubrimientos (Abreu 2015; Vaz-Canosa y Rodríguez Cajarville 2015; Grattarola et al., 2016; Rocha, 2019; Serra Alanis, Scarabino, Wlodek, Furtado y Balao, 2020), redundando entre otros aspectos en el aumento del número de especies reconocidas en varios taxa. Si bien esta información es reconocida y accesible dentro del ámbito científicoacadémico, muchas veces no llega a conocerse por el resto de la población.

El objetivo del presente trabajo es evaluar el estado actual del conocimiento de la fauna de vertebrados tetrápodos autóctonos en las personas que visitan el Museo de Historia Natural Dr. Carlos A. Torres de la Llosa (dependiente de la Dirección General de Educación Secundaria).

\section{MATERIALES Y MÉTODOS}

Para evaluar el conocimiento sobre especies autóctonas se realizó una encuesta semi-estructurada (Cea D'Ancona, 1996) entre los grupos de visitantes del Museo de Historia Natural Dr. Carlos A. Torres de la Llosa previo a recorrer sus instalaciones. Se encuestó un total de 593 personas durante los años 2015 y 2016: docentes, estudiantes de enseñanza media y terciaria de Montevideo e interior del país con un rango de edad entre 12 y 60 años. La misma constaba de dos partes: la primera se solicitaba escribir de memoria el nombre (nombre común) de cinco especies de cada grupo de vertebrados tetrápodos (mamíferos, aves, reptiles y anfibios); para lo cual contaban con 15 minutos. La segunda instancia constaba en observar 16 fotografías de mamíferos autóctonos durante 30 segundos cada una y nombrar la especie visualizada (también nombre común), dichos especies eran las siguientes: Ctenomys pearsoni tucutucu, Hydrochoerus hydrochaeris carpincho, Myocastor coypus nutria, Cavia aperea apereá, Cerdocyon thous zorro de monte, Panthera onca yaguareté, Puma concolor puma, Conepatus chinga zorrillo, Galictis cuja huron, Lontra longicaudis lobito de río, Nasua nasua coatí, Pecarí tajacu pecarí de collar, Ozotoceros bezoarticus venado de campo, Mazama gouazoubira guazubirá, Tamandúa tetradactyla tamandúa y Dasypus hybridus mulita.

Los encuestados se agruparon por nivel de formación: Secundaria $(n=501)$ Terciaria $(n=92)$, lugar de origen: Montevideo $(n=342)$ Interior $(n=208)$ y edad: menores $(n=384)$ y adultos $(n=209)$. 


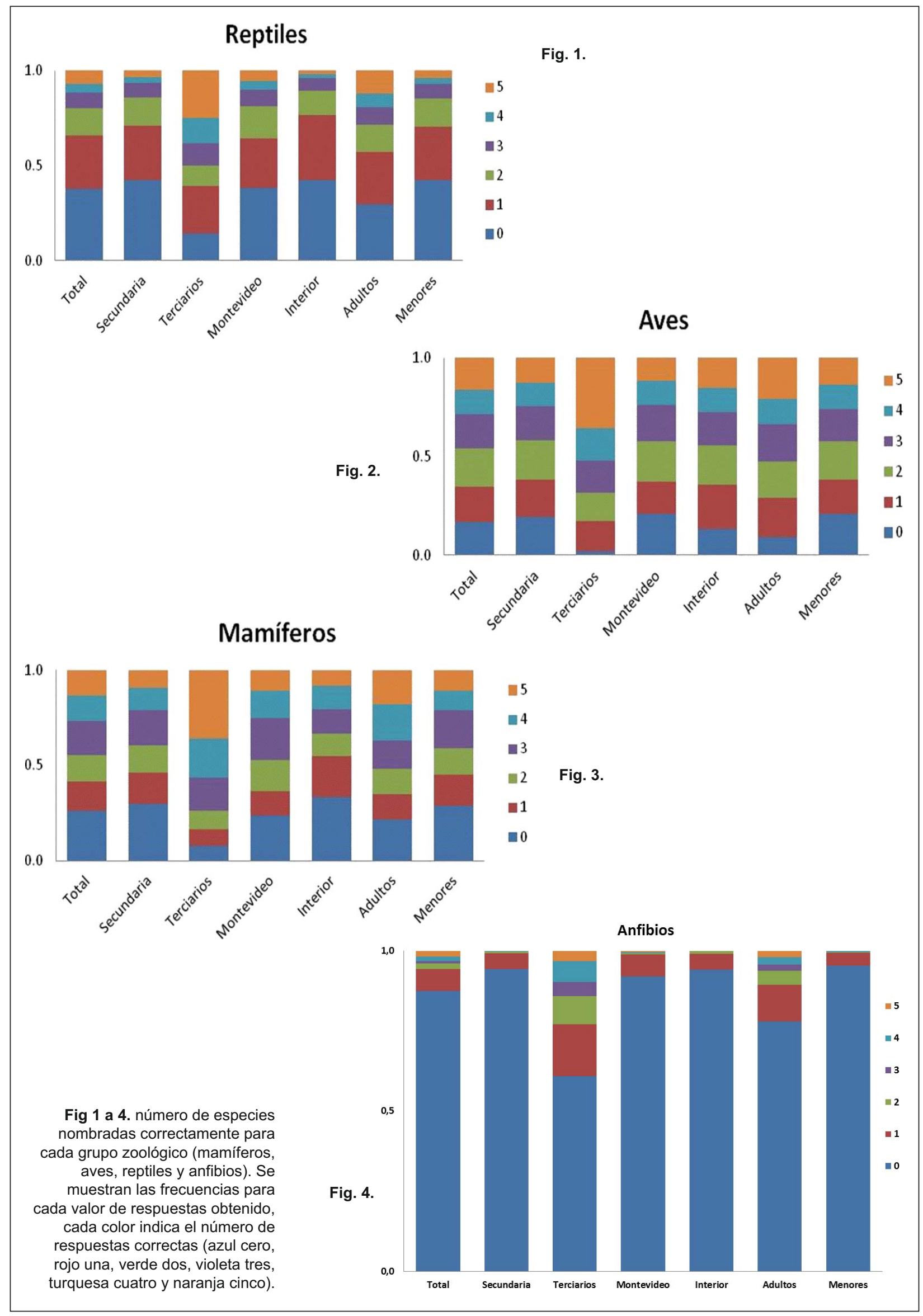

Bol. Soc. Zool. Uruguay (2a época). 2021. Vol. 30 (1): 1-13 ISSN 2393-6940 


\section{RESULTADOS}

Las Fig. 1 a 4 muestran la frecuencia de respuestas correctas de cada grupo zoológico discriminada por total de participantes y por cada subgrupo en que se dividió la muestra. En las mismas podemos observar que a nivel de todos los encuestados quienes lograron mencionar espontáneamente de forma correcta a cinco especies mostraron frecuencias de 0,162 (aves), 0,135 (mamíferos), 0,067 (reptiles) y 0,018 (anfibios); siendo este último el grupo que mayor frecuencia mostró de personas que no lograron nombrar ninguna especie de forma correcta $(0,875)$, seguido de reptiles $(0,378)$, mamíferos $(0,263)$ y aves $(0,166)$. Esta tendencia indica que las aves son el grupo que más se conoce y anfibios el que menos se observa dentro de los diferentes grupos en los que se dividió la muestra.

Las personas con estudios terciarios fueron el grupo que mejores resultados exhibió al responder espontáneamente las cinco especies de cada grupo zoológico solicitada obteniendo frecuencias de 0,359 (aves y mamíferos), 0,250 (reptiles) y 0,033 (anfibios) a la vez que mostraron los resultados más bajos en cuanto a ninguna especie mencionada correctamente: 0,022 (aves), 0,076 (mamíferos), 0,141 (reptiles) y 0,609 (anfibios). Los estudiantes de secundaria exhibieron valores de 0,125 (aves), 0,092 (mamíferos), 0,033 (reptiles) y 0,000 (anfibios) para cinco especies nombradas correctamente.

Si tomamos en cuenta la diferenciación geográfica, personas que viven en la capital del país (Montevideo) respecto a quienes viven en localidades del interior del país (fuera de la capital) los resultados se muestran muy parejos en cuanto a las cinco especies mencionadas correctamente: 0.110-0.082 (mamíferos); 0,116-0,154 (aves); 0,052-0,019 (reptiles) y 0,003-0,000 (anfibios), en todos los casos el primer valor corresponde a los habitantes de Montevideo. En cuanto a ninguna especie nombrada de forma correcta los resultados obtenidos fueron los siguientes: 0,238-0,332 (mamíferos), 0,207-0,130 (aves), 0,384-0,423 (reptiles) y 0,920-0,942 (anfibios), también en este caso el primer valor corresponde a Montevideo.

Los adultos también mostraron una tendencia a obtener mejores resultados que los menores de 18 años de edad. Los resultados al momento de nombrar cinco especies correctas fueron (adultos primero): 0,182-0,108 (mamíferos), 0,206-0,138 (aves), 0,1200,038 (reptiles) y 0,019-0,000 (anfibios). En cuanto a cero especies mencionadas correctamente los resultados fueron 0,215-0,289 (mamíferos), 0,0910,208 (aves), 0,297-0,424 (reptiles) y 0,780-0,954 (anfibios).

Las especies correcta e incorrectamente nombradas de cada grupo zoológico junto con el número de veces que fueron mencionadas por los encuestados se muestran en las tablas 1 y 2 respectivamente. Uno de los aspectos más destacables es que las especies mayormente nombradas de forma correcta fueron las que figuran en las monedas de circulación corriente (Carpincho, Mulita, Ñandú, Puma) y las erróneamente mencionadas fueron especies de interés productivo, mascotas o especies que habitan ambientes antropizados.

Respecto a la identificación de las especies a partir de las imágenes las frecuencias de respuestas correctas se muestran en la Tabla 3. En este caso podemos observar que las especies que mayor frecuencia obtuvieron fueron especies comunes de ver en nuestro territorio además de las mencionadas que figuran en las monedas (Zorrillo, Zorro gris, Carpincho, Mulita, Puma).

En la Fig. 5 se observa el número total de imágenes identificadas correctamente. En la misma se puede notar que la mayoría de las respuestas se ubican en el rango de cinco a diez imágenes correctamente identificadas siendo siempre en valores inferiores a 0.250 .

\section{DISCUSIÓN}

El presente trabajo es una aproximación general del conocimiento que los uruguayos poseen sobre la fauna autóctona. Si bien los datos no son de una muestra aleatoria, ni estandarizada sino que fueron los visitantes al museo, por lo que puede contener sesgos, de todas formas consideramos que los resultados obtenidos pueden ser un insumo de partida para futuras investigaciones y propuestas. A su vez pueden existir posibles interferencias respecto a nombres comunes que signifiquen especies diferentes en las distintas personas, pero siendo público no especializado lo importante en este caso eran las referencias comunes en la población y no del público especializado en el tema.

En una primera instancia los resultados obtenidos reflejan un conocimiento bastante escaso de nuestra fauna. Este hecho concuerda con lo planteado previamente por Pérez-Miles (1996) en un estudio realizado con escolares en Uruguay. Este desconocimiento general no es algo que pueda atribuirse solamente a la población de Uruguay ya que la misma tendencia se ha encontrado en otras latitudes. A modo de ejemplo Paraskevopoulos, Padeliadu y Zafiropoulos (1998) con estudiantes griegos de 5 to y 6 to año de primaria, concluyen que los niños tienen un menor interés en los seres vivos silvestres, lo que no pueden explicar el pobre conocimiento sobre especies nativas. A su vez estudios realizados sobre el conocimiento de estudiantes sobre el bioma "Cerrado" de Brasil, encontraron que los participantes no podían identificar un número importante de animales nativos de Brasil (Bizerril y Andrade, 1999; Bizerril, 2004). Misma 
Tabla 1: especies nombradas correctamente por los encuestados. Se observa la riqueza total por grupo zoológico y el número de veces que fueron mencionadas.

\begin{tabular}{|c|c|c|c|c|c|c|c|}
\hline \multicolumn{2}{|l|}{$\begin{array}{l}\text { MAMIFEROS } \\
\quad(\alpha=38)\end{array}$} & \multicolumn{2}{|l|}{$\begin{array}{c}\text { AVES } \\
(\alpha=88)\end{array}$} & \multicolumn{2}{|l|}{$\begin{array}{l}\text { REPTILES } \\
(\alpha=24)\end{array}$} & \multicolumn{2}{|l|}{$\begin{array}{c}\text { ANFIBIOS } \\
(\alpha=23)\end{array}$} \\
\hline Carpincho & 345 & Tero & 333 & Yacaré & 159 & Sapito Darwin & 31 \\
\hline Mulita & 248 & Ñandú & 213 & Lagarto & 155 & Cururú & 20 \\
\hline Puma & 135 & Hornero & 146 & Crucera1 & 16 & Escuerzo & 10 \\
\hline Tatú & 96 & Cardenal & 109 & Yarará & 99 & Rhinella arenarun & 9 \\
\hline Gato montés & 84 & Benteveo & 49 & Lagartija & 60 & Cecilia & 6 \\
\hline Nutria & 75 & Cotorra & 48 & Cascabel & 41 & Rana monito & 6 \\
\hline Venado de campo & 65 & Chajá & 43 & Coral & 34 & Rana gato & 4 \\
\hline Zorro & 58 & Gaviota & 39 & Morrocoyo & 26 & Rana trepadora & 4 \\
\hline Apereá & 53 & Carpintero & 28 & Parejera & 12 & Rana gato & 3 \\
\hline Tucutucu & 49 & Perdiz & 23 & Falsa coral & 10 & Rana boyadora & 2 \\
\hline Zorrillo & 41 & Calandria & 20 & Falsa crucera & 10 & Rana verde & 2 \\
\hline Coatí & 31 & Carancho & 19 & Tortuga campanita & 7 & Sapito de jardín & 2 \\
\hline Comadreja & 31 & Golondrina & 19 & Tortuga cuello de víbora & 6 & Bufo Dorbigni & 1 \\
\hline Yaguareté & 31 & Gallineta & 18 & Tortuga canaleta & 4 & Escuerzo chico & 1 \\
\hline Guazubirá & 28 & Churrinche & 17 & Víbora ciega & 4 & Rana común & 1 \\
\hline Mano pelada & 18 & Colibrí & 17 & Víbora de dos cabezas & 4 & Rana criolla & 1 \\
\hline Lobo marino & 17 & Zorzal & 15 & Culebra verde & 2 & Rana roncadora & 1 \\
\hline Lobito de río & 10 & Águila mora & 14 & Musurana & 2 & Ranita de cuatro ojos & 1 \\
\hline Zorro gris & 8 & Tijereta & 12 & Tortuga verde & 2 & Ranita Zarzal & 1 \\
\hline Aguara guazú & 7 & Torcaza & 12 & Lagartija de la arena & 1 & Sapito de Fernández & 1 \\
\hline Oso hormiguero & 7 & Flamenco & 11 & Tortuga 7 quillas & 1 & Sapito verde & 1 \\
\hline Ballena franca & 6 & Cisne cuello negro & 9 & Tortuga cabezona & 1 & Sapo de jardín & 1 \\
\hline Murciélago & 6 & Tordo & 9 & Tortuga carey & 1 & Sapo de la arena & 1 \\
\hline Coendú & 5 & Dorado & 7 & Tortuga herradura & 1 & & \\
\hline Tatú carreta & 4 & Gavilán & 7 & & & & \\
\hline Huron & 3 & Martin pescador & 7 & & & & \\
\hline Lobo fino & 3 & Chingolo & 6 & & & & \\
\hline Ratón de campo & 3 & Garza & 6 & & & & \\
\hline Lobo peluca & 2 & Garza mora & 6 & & & & \\
\hline Ballena franca & 1 & Sabia & 5 & & & & \\
\hline
\end{tabular}

tendencia se encontró en niños ingleses (Huxham, Welsh, Berry y Templeton, 2006) y en España y Portugal (Almeida, García Fernández y StrechtRibeiro, 2018), estos últimos autores concluyeron que los niños sabían más de los animales de la Sabana africana que de los animales ibéricos. Resultados similares se encontraron en estudios que involucraban a estudiantes de formación docente de diferentes países europeos (Lindemann-Matthies, Constantinou,
Lehnert, Nagel, Raper y Kadji-Beltran, 2011; Sánchez Emeterio y García Fernández, 2013) y en Colombia (Zapata-Ciro, Guevara y Castaño-Villa, 2016; Salas 2018). Sin embargo, otros estudios han reportado, al menos de forma parcial, resultados opuestos. Este es el caso del estudio de Patrick y Tunnicliffe (2011) con una muestra de niños ingleses y estadounidenses: el primer grupo mencionó más animales exóticos y el segundo más nativos, lo que abre la posibilidad de 
Tabla 2. especies nombradas incorrectamente por los encuestados. Se observa la riqueza total por grupo zoológico y el número de veces que fueron mencionadas.

\begin{tabular}{|c|c|c|c|c|c|c|c|}
\hline \multicolumn{2}{|l|}{$\begin{array}{l}\text { Mamíferos } \\
\quad(\alpha=53)\end{array}$} & \multicolumn{2}{|c|}{$\begin{array}{c}\text { Aves } \\
(\alpha=25)\end{array}$} & \multicolumn{2}{|l|}{$\begin{array}{l}\text { Reptiles } \\
(\alpha=44)\end{array}$} & \multicolumn{2}{|l|}{$\begin{array}{l}\text { Anfibios } \\
(\alpha=57)\end{array}$} \\
\hline Vaca & 92 & Paloma & 69 & Serpientes & 39 & Rana & 116 \\
\hline Perro & 57 & Gorrión & 51 & Tortuga & 39 & Sapo & 109 \\
\hline Gato & 44 & Avestruz & 39 & Culebra & 28 & Renacuajo & 8 \\
\hline Jabalí & 43 & Gallina & 17 & Iguana & 28 & Bagre & 7 \\
\hline Caballo & 34 & Faisán & 12 & Cocodrilo & 27 & Carpincho & 7 \\
\hline Oveja & 34 & Loro & 9 & Vibora & 25 & Lobo marino & 5 \\
\hline Liebre & 25 & Pájaro & 8 & Rana & 12 & Lombriz & 5 \\
\hline Ñandú & 23 & Canario & 6 & Caiman & 8 & Serpiente & 5 \\
\hline Chancho & 19 & Pato & 6 & Salamandra & 7 & Araña & 4 \\
\hline Conejo & 15 & Ganso & 4 & Sapo & 6 & Lagartija & 4 \\
\hline Cabra & 12 & Pavo & 4 & Lombriz & 5 & Lagarto & 4 \\
\hline Ciervo & 9 & Pollo & 4 & Saltamontes & 5 & Tortuga & 4 \\
\hline Perro cimarrón & 9 & Murciélago & 3 & Serpiente de maíz & 5 & Mojarra & 3 \\
\hline Humano & 7 & Codorniz & 2 & Araña pollito & 4 & Nutria & 3 \\
\hline León & 7 & Guinea & 2 & Boa & 4 & Salamandra & 3 \\
\hline Mono & 7 & Papagayo & 2 & Murciélago & 4 & Vibora & 3 \\
\hline Tigre & 7 & Pavo real & 2 & Araña & 3 & Ballena & 2 \\
\hline Cordero & 5 & Tucutucu & 2 & Cobra & 3 & Cabeza amarga & 2 \\
\hline Rata & 5 & Coatí & 1 & Liebre & 3 & Cangrejo & 2 \\
\hline Toro & 5 & Cóndor & 1 & Tarántula & 3 & Lisa & 2 \\
\hline Cui & 4 & Guazubirá & 1 & Carpincho & 2 & Medusa & 2 \\
\hline Llama & 4 & Hámster & 1 & Hormiga & 2 & Peces & 2 \\
\hline Ratón & 4 & Mulita & 1 & Mulita & 2 & Pez payaso & 2 \\
\hline Cebra & 3 & Nutria & 1 & Tatú & 2 & Tararira & 2 \\
\hline Perrito de las praderas & 3 & Plumerillo & 1 & Viboras & 2 & Yacaré & 2 \\
\hline Castor & 2 & & & Apereá & 1 & Abeja & 1 \\
\hline Cimarrón & 2 & & & Babosa & 1 & Anguila eléctrica & 1 \\
\hline Elefante & 2 & & & Cabra & 1 & Caballito de mar & 1 \\
\hline Foca & 2 & & & Calamar & 1 & Caballo & 1 \\
\hline Jirafa & 2 & & & Camaleón & 1 & Cangrejo de río & 1 \\
\hline
\end{tabular}

diferentes resultados de acuerdo al contexto cultural por lo que sería otro aspecto a tener en cuenta en futuros estudios sobre la temática.

Un aspecto en el que podría profundizarse a futuro es evaluar como difiere el conocimiento de la fauna autóctona respecto al sexo y la edad de la población.

En cuanto al sexo no abundan los trabajos que tengan este enfoque, al caso podemos citar a Seger, Solé, Martínez y Schiavetti (2019) en una investigación sobre anfibios en la Mata Atlántica mencionan que los hombres reconocieron más especies que las mujeres. Lo mismo se encontró respecto al conocimiento de aves en el Noreste de Brasil (Vieira Santos, Medeiros Silva Souto, Serejo Neves Ribeiro, Farias Paiva de Lucena y Guzzi, 2020).

Respecto a la edad en el presente trabajo se observó una tendencia a que los adultos conocen más a la fauna nativa que los menores de edad. Sin 


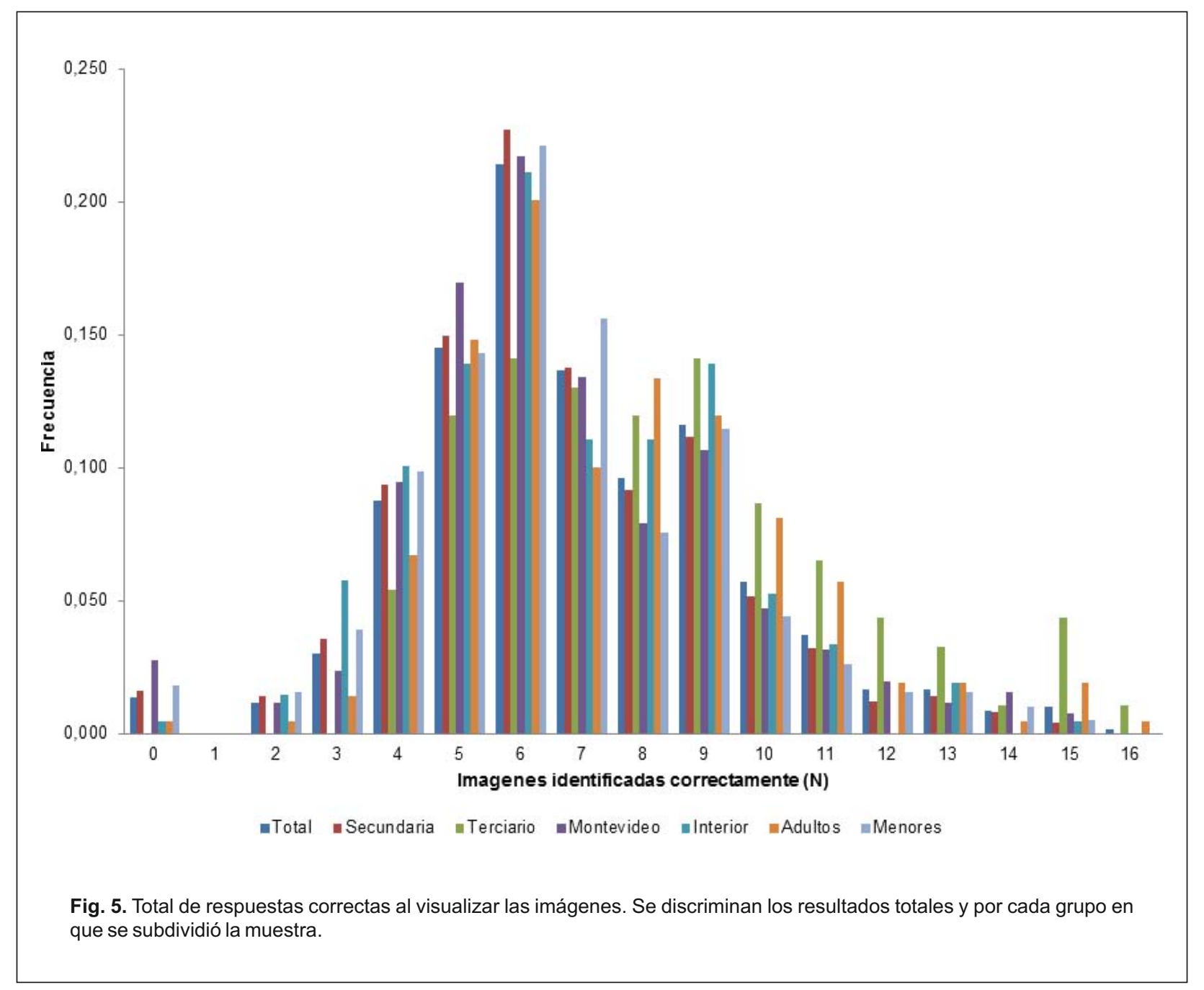

embargo los resultados que presentaron otras investigaciones son diversos y no se puede observar una tendencia única al respecto. Huxham, et al. (2006) encontraron en el Reino Unido que el conocimiento sobre diferentes especies aumenta con la edad, llegando a su máximo a los nueve años, decayendo un poco luego. Dicha tendencia también fue hallada en estudiantes alemanes por Randler (2008) pero el máximo fue a los 14 años de edad y se relacionó este patrón con la disminución en el interés sobre la biología durante la pubertad. Aun así, un estudio posterior de este autor encontró que el declive generacional del interés por la zoología durante las últimas tres décadas fue residual en comparación con el de la botánica (Randler, Osti y Hummel, 2012). Asimismo una tendencia similar fue encontrada en estudios enfocados en aves en estudiantes eslovacos de entre 10 y 19 años de edad, que concluyó que los más jóvenes tienen más conocimiento sobre esos animales que los mayores (Prokop, Kubiatko, y
Fančovičová, 2008), en otro trabajo con estudiantes de primaria con una edad promedio de 12.3 y estudiantes universitarios de biología con un promedio de 21.2 (Prokop y Rodak 2009), a su vez en Costa Rica se halló la misma tendencia (Guido y Rodríguez 2015).

Asimismo, estudios llevados adelante en Escocia observaron que los niños de entre cuatro y 12 años de edad son quienes tienen un mayor conocimiento de mamíferos que de aves e invertebrados. (Huxham et al, 2006) esto también fue encontrado en estudiantes universitarios de Inglaterra (Batt 2009). Esto mismo fue hallado por Patrick et al. (2013) en un trabajo llevado a cabo en seis países (Brasil, Inglaterra, Finlandia, Islandia, Portugal y Estados Unidos) halló que los estudiantes de diferentes edades: seis, 10 y 15 , dada la tarea de nombrar tantos animales como fuera posible, estaban más conscientes de los mamíferos como ejemplos. Estos resultados se explicaron por la presencia de mamíferos en medios 
Tabla 3. Número absoluto de respuestas correctas por encuestados al visualizar las imágenes. Se discriminan los resultados totales y por cada grupo en que se subdividió la muestra. Número total de encuestas: 593.

\begin{tabular}{lccccccc} 
Especie & $\begin{array}{c}\text { Total } \\
\text { correctas }\end{array}$ & $\begin{array}{c}\text { Secundaria } \\
(\mathbf{n}=501)\end{array}$ & $\begin{array}{c}\text { Terciaria } \\
(\mathbf{n}=\mathbf{9 2})\end{array}$ & $\begin{array}{c}\text { Montevideo } \\
(\mathbf{n}=\mathbf{3 4 2})\end{array}$ & $\begin{array}{c}\text { Interior } \\
(\mathbf{n}=\mathbf{2 0 8})\end{array}$ & $\begin{array}{c}\text { Adulto } \\
(\mathbf{n}=\mathbf{2 0 9})\end{array}$ & $\begin{array}{c}\text { Menores } \\
(\mathbf{n}=\mathbf{3 8 4})\end{array}$ \\
\hline Tucutucu & 68 & 44 & 24 & 22 & 26 & 35 & 33 \\
Carpincho & 523 & 437 & 86 & 301 & 180 & 193 & 330 \\
Nutria & 178 & 133 & 45 & 83 & 65 & 81 & 97 \\
Apereá & 211 & 172 & 39 & 109 & 80 & 96 & 115 \\
Zorro & 530 & 444 & 86 & 297 & 192 & 189 & 341 \\
Yaguareté & 182 & 139 & 43 & 111 & 47 & 76 & 106 \\
Puma & 469 & 399 & 70 & 291 & 146 & 158 & 311 \\
Zorrillo & 560 & 472 & 88 & 322 & 197 & 198 & 362 \\
Huron & 213 & 182 & 31 & 126 & 68 & 63 & 150 \\
Lobito de río & 79 & 47 & 32 & 29 & 28 & 49 & 30 \\
Coatí & 151 & 120 & 31 & 86 & 47 & 53 & 98 \\
Pecarí & 42 & 25 & 17 & 19 & 9 & 21 & 21 \\
Venado de campo & 186 & 147 & 39 & 98 & 62 & 76 & 110 \\
Guazubirá & 58 & 46 & 12 & 35 & 14 & 27 & 31 \\
Tamandúa & 167 & 138 & 29 & 96 & 55 & 46 & 121 \\
Mulita & 529 & 444 & 85 & 308 & 180 & 192 & 337 \\
\hline
\end{tabular}

de comunicación, juguetes y libros. A su vez su presencia refleja el hecho de que en general son más grandes que otros animales y pueden fácilmente llamar la atención humana. Este resultado de que los mamíferos y luego las aves son el grupo más conocido coincide con lo observado en nuestro país.

Otro de los aspectos a profundizar respecto al conocimiento de fauna por parte de la población es evaluar como varía el mismo según el origen geográfico o el entorno urbano-rural. Si bien aquí se observaría una tendencia a conocer más por parte de los residentes en Montevideo no podemos ser concluyentes porque la población de estudio que provenía del interior del país era de centros urbanos y no de población rural. En Uruguay la población rural es cercana al 4\% (INE 2014) lo que dificulta por el simple número, tener acceso a dicha población. Estudios en varios países han revelado diferencias en el conocimiento y la percepción existente respecto a la fauna local. En este sentido Redford y Robinson (1991) y Zuñiga (1994) sostienen que en comunidades rurales $\mathrm{O}$ indígenas, los pobladores que presentan mayor conocimiento sobre la vida silvestre son aquéllos que han tenido una relación directa con el campo. Sin embargo, este conocimiento es superado para especies con importancia cinegética.
Seger et al (2019) encontraron también un vínculo lejano de los pobladores de la Mata Atlántica con los anfibios, a pesar del registro de cierto nivel de conciencia ecológica, evidente cuando los pobladores declararon no matar ranas porque comen insectos o están asociados con la mala suerte. Esto último también lo halló González Hernández (2019) en pobladores rurales del Estado de México, a su vez menciona que las especies más comunes son las más conocidas y las que tienen cierta importancia alimenticia, medicinal o mágico-religiosa.

En cuanto al nivel educativo los resultados obtenidos estrían mostrando cierta influencia positiva en cuanto al conocimiento de la fauna nativa lo cual estaría en concordancia con parte de lo planteado por Randler (2010) quien encontró que el conocimiento de especies nativas está correlacionado con la edad, nivel de escolaridad, frecuencia de caminatas en la naturaleza, leer libros temáticos o visitar parques de juegos. Sin embargo existen resultados que tienden a contradecir lo anterior. Louv (2010) afirmó que la escuela no revierte la tendencia de no conocer las especies nativas, ya que los niños saben mucho sobre la selva tropical y sus amenazas pero no saben nada sobre sus propios bosques. Con frecuencia, ni siquiera conocen los campos cercanos a la escuela 
que ofrecen tantas formas de vida que podrían ser estudiados. Sin embargo, en una investigación desarrollada en Costa Rica sobre el conocimiento de aves se encontró una correlación positiva significativa entre escolaridad y especies reconocidas (Guido y Rodríguez 2015). Similares resultados se obtuvieron en España y Portugal donde los estudiantes de magisterio fueron mucho mejores en identificar animales ibéricos que los estudiantes de niveles iniciales (Almeida et al., 2019). Estos autores plantean que las dificultades de los niños con la identificación de animales ibéricos también pueden estar relacionadas con la falta de acercamiento a las especies autóctonas en la escuela, debido a que no existe referencia en los planes de estudio de los países a la importancia de la fauna nativa (Almeida et al 2019). En este sentido, Vieira Santos et al (2020) mencionan que el nivel educativo también influyó en el conocimiento de aves locales en el Noreste de brasileño.

\section{CONCLUSIONES}

El presente trabajo tuvo como objetivo evaluar el conocimiento existente respecto a la fauna autóctona en los asistentes a las visitas guiadas del Museo de Historia Natural Dr. Carlos A. Torres de la Llosa. Si bien en líneas generales se puede decir que dicho conocimiento es escaso, esto permite abrir futuras preguntas en las que se investiguen sus causas. Se desprende la necesidad de realizar más investigaciones en otros contextos (urbano o rural), niveles educativos y países, para identificar si los patrones encontrados son exclusivos del contexto estudiado, o si también se pueden encontrar en otros marcos (Almeida et al, 2019).

Diversos autores destacan la disminución del contacto con la naturaleza en todo el mundo, que se ha acrecentado en las últimas décadas, especialmente entre la generación urbana más joven como el de mayor importancia al momento de conocer a las especies nativas (Clavero y De Los Reyes 2003; Kellert, 2005; Eshach 2006; Pergams y Zaradic 2006; Gelman 2009; Patrick y Tunnicliffe, 2011; Heezik, Dickinson y Freeman, 2012; Mateos Jiménez y García Fernández, 2016; Soga y Gaston, 2016; Almeida et al., 2018; White, Eberstein y Scott 2018), lo que puede ser un punto de partida a futuro para realizar futuros estudios.

Como forma de generar un mayor conocimiento de la fauna local existe la idea de que visitar lugares silvestres donde exista una mayor conexión con la fauna y la flora locales puede mejorar el conocimiento y el interés de los niños por la naturaleza, principalmente desde la infancia y continuando en la adolescencia, entre las personas jóvenes con la naturaleza y el ambiente inmediato en el que se desarrollan (Clavero y De Los Reyes 2003; Eshach
2006; Pergams y Zaradic 2006; Gelman 2009; Patrick y Tunnicliffe, 2011; Heezik, Dickinson, y Freeman, 2012; Mateos Jiménez y García Fernández, 2016; Almeida et al., 2018; White, Eberstein y Scott 2018).

Independientemente de los resultados de cada país o región uno de los factores que parece influir es la educación. Para esto se deberían promover planes educativos en todos los niveles que estimulen el conocimiento de nuestra fauna y flora autóctona. La creación y promoción de programas de educación y formación para estudiantes, profesionales, técnicos y la comunidad académica en general es imprescindible para lograr aproximarse al conocimiento de la diversidad biológica (Gasca Álvarez y Torres Rodríguez 2013; Almeida et al 2019).

También varios autores mencionan la ignorancia respecto a la fauna autóctona con la influencia de los medios de comunicación que divulgan principalmente animales exóticos a través de dibujos animados, películas, juegos, libros infantiles y documentales que, frecuentemente, destacan a los grandes mamíferos africanos lo que genera que en la vida cotidiana se haga referencia a dichos animales (Scalfi, 2014; Torres et al., 2018; Bermeo 2020).

Otro aspecto importante a tener en cuenta es el fortalecimiento de la capacidad divulgativa de los centros e institutos, que basados en el conocimiento generado, cumplen la función de comunicar a la comunidad el trabajo realizado y las decisiones concertadas que favorezcan tanto a grupos ecológicos como humanos. Así, las estrategias de conservación dejan de interesar sólo a comunidades científicas para convertirse en una actividad social, en donde es necesario que participen todas y cada una de las personas que poseen un entorno natural y que se benefician de una u otra forma de éste (Gasca Álvarez y Torres Rodríguez, 2013). Dichas acciones de divulgación que llevan al conocimiento de la ciencia $y$, en este contexto, sobre la biodiversidad y la necesidad de preservarla, también pueden ser vistas como herramientas de inclusión social y no sólo como instrumentos de difusión de la información (Scalfi et al., 2016). Sobre esto Kareiva y Marvier (2012) mencionan la importancia de tener en cuenta la psicología humana y el impacto de los mensajes en las personas y que una de las estrategias para aumentar el apoyo al conocimiento de la naturaleza y su conservación debe centrarse en los niños y adolescentes para volver a conectarlos con la naturaleza. Para este fin, pueden emplearse estrategias sencillas como la difusión de publicaciones pedagógicas, con un lenguaje sencillo y ameno que permita acercar el conocimiento a la población (Gasca Álvarez y Torres Rodríguez, 2013). Asimismo, se pueden generar estrategias múltiples para la divulgación como libros de cuentos (Huxham 2006; Bermeo, 2020), TICs (Aguilera, Gómez y Gutiérrez, 2015, Sepúlveda-Peña et al., 2019), medios audiovisuales con foco en nuestra fauna o hasta 
elementos que ajenos a ese fin logran un resultado no esperado pero positivo como el dinero circulante (Scalfi et al., 2016). En este trabajo las especies más nombradas son las que contienen las monedas de circulación corriente. Sin embargo, no es posible determinar que esta sea la causa o que puedan haber sido elegidas como íconos monetarios por ser especies ampliamente conocidas por la población; la elección de usar animales o plantas nativas en el dinero se ha dado en países como Brasil, Sudáfrica, Venezuela, Argentina y Bielorrusia, que poseen una enorme biodiversidad, optaron en algún momento de su trayectoria, mostrar la naturaleza nativa en sus billetes, por medio de animales y plantas (Scalfi et al., 2016). Todos estos elementos son una fuente a través de la cual es probable que las personas pueden recibir información sobre la vida silvestre y son una forma posible de ayudar con la popularización, difusión y mejora de conocimientos relacionados con especies autóctonas, ya que no siempre es posible el contacto y la experiencia con animales salvajes en vivo (Bermeo, 2020).

Deberían pensarse estrategias amplias y abarcativas para poder generar un mayor conocimiento de la fauna autóctona en la población uruguaya. Lo que no se conoce difícilmente va a ser conservado, la falta de apropiación y empoderamiento por los recursos faunísticos locales y nacionales, generan un desinterés por la preservación de las especies (Bermeo, 2020).

\section{AGRADECIMIENTOS}

Quiero agradecer a quienes de alguna u otra forma aportaron a la realización de este trabajo. A mis compañeros del Museo de Historia Natural Dr. Carlos A. Torres de la Llosa en especial a Jacquelinne Prochet y Víctor G. Bacchetta, a Inés Campanella, Fernando Pérez-Miles, Enrique M. González, Diego Procopio, Analía Caballero y a todos los participantes de la encuesta.

\section{BIBLIOGRAFÍA}

Abreu M. (2015). Primer registro de gaviota de Franklin Leucophaeus pipixcan (Wagler, 1831) (Aves: Laridae) en Uruguay. Boletin de la Sociedad Zoologica del Uruguay (2a época), 24(1): 22-27.

Aguilera, A., Gómez C., y Gutiérrez, M. (2015). Conozcamos lo mejor de la flora y fauna de la región del Ariari en el municipio de Granada, departamento del Meta. "Catarsis turística." Repositorio Fundación Universitaria Los Libertadores, 53(9), 1689-1699.

Almeida, A., García Fernández, B., y Strecht-Ribeiro, O. (2018). Children's knowledge and contact with native fauna: A comparative study between Portugal and Spain. Journal of Biological Education, 54(1), 17-32.

Almeida, A., García Fernández, B., y Stretch-Ribeiro, O. (2019). Primary school children and preservice teachers' knowledge of iberian native and african savannah mammals. Journal of Baltic Science Education, 18(6), 833-847.

Arias Rodríguez, A., Torralba-Burrial, A., Anadón Álvarez, M.A., Lastra López, C.G., García Albá, J., y Herrero Vázquez, M. (2018). Desarrollo de una Colección Virtual de Fauna Asturiana: una herramienta innovadora en la Didáctica de la Zoología. XI Jornadas de Innovación Docente 2018 Innovar la docencia hacia la igualdad de género. Creando espacios para compartir experiencias. XI JORNADAS DE INNOVACIÓN DOCENTE 2018 - Libro de Actas Universidad de Oviedo, 90-102.

Bath, A.J. (1996). Increasing the applicability of human dimensions research to large predators. Journal of Wildlife Research, 1: 215-220.

Batt, S. (2009). Human attitudes towards animals in relation to species similarity to humans: A multivariate approach. Bioscience Horizons: The International Journal of Student Research, 2(2), 180-190.

Ballouard, J.M., Brischoux, F., y Bonnet, X. (2011). Children prioritize virtual exotic biodiversity over local biodiversity. PloS ONE, 6(8), e23152

Barrera, B.N., y Toledo, V. (2005). Ethnoecology of the Yucatec maya: Symbolism, knowledge and management of natural resources. Journal of Latin American Geography, 4: 9-41.

Bermeo, M L. (2020). Libro de cuentos para niños de fauna nativa colombiana, como estrategia educativa de aprendizaje y conservación de especies. Trabajo presentado para obtener el título de Especialista en Educación Ambiental. Fundación Universitaria Los Libertadores Facultad de Ciencias Humanas y Sociales. Bogotá D.C.

Bizerril, M., y Andrade, T (1999). Knowledge of the urban population about fauna: Comparison between Brazilian and exotic animals. Ciência e Cultura: Journal of The Brazilian Association for the Advancement of Science, 51(1), 38-41.

Bizerril, M. (2004). Children's perceptions of Brazilian Cerrado landscapes and biodiversity. The Journal of Environmental Education, 35(4), 4758.

Cea D'Ancona, M.A. (1996). Metodología Cuantitativa: Estrategias y Técnicas de Investigación Social. Síntesis.

Clavero, M., y De Los Reyes, L. (2003). Nombres y conocimiento popular de las aves en Benaocaz (Cádiz). Revista de la sociedad gaditana de historia natural. 3, 215-226.

Eshach, H. (2006). Science literacy in primary and pre- 
schools. Dordrecht (The Netherlands): Springer.

Gasca Álvarez, H.J., y Torres Rodríguez, D. (2013). Conservación de la biodiversidad en Colombia, una reflexión para una meta: conocer y educar para conservar. Cuadernos de Biodiversidad 42, 31-37.

Gelman, S.A. (2009). Learning from others: Children's construction of concepts. Annual Review of Psychology, 60, 115-140.

Genovart, M., Tavecchia, G., Enseñat, J.J., y Laiolo, P. (2013). Holding up a mirror to the society: Children recognize exotic species much more than local ones. Biological Conservation, 159, 484-489.

Glowka, L., Burhenne-Guilmin, F., y Synge, H. (1994). Guide to the convention on biological diversity. Gland and Cambidge: IUCN, $161 \mathrm{pp}$

González, E.M., y Martínez-Lanfranco, J.A. (2010). Mamíferos de Uruguay. Guía de campo e introducción a su estudio y conservación. Vida Silvestre-Museo Nacional de Historia Natural. Ediciones de la Banda Oriental, Montevideo. $462 \mathrm{pp}$.

González Hernández, A. (2019). Estudio etnozoológico de anfibios en el estado de México. Universidad autónoma del estado de México Tesis de Maestría.

Grattarola, F., Hernández, D., Duarte, A., Gaucher, L., Perazza, G., González, S., ... Rodriguez-Tricot, L. (2016). Primer registro de yaguarundí (Puma yagouaroundi) (MAMMALIA: CARNIVORA: FELIDAE) en Uruguay, con comentarios sobre monitoreo participativo. Boletin de la Sociedad Zoologica del Uruguay (2a época), 25(1), 85-91.

Guido, I., y Rodríguez, C. (2015). Conocimiento popular sobre las aves de Costa Rica. Zeledonia, 19:1, 3-9.

Heezik, Y., Dickinson, K., y Freeman, C. (2012). Closing the gap: Communicating to change gardening practices in support of native biodiversity in urban private garden. Ecology and Society, 17(1), 34.

Huxham, M., Welsh, A., Berry, A., y Templeton, S. (2006). Factors influencing primary school children's knowledge of wildlife. Journal of Biological Education, 41(1), 9-12.

INE. 2004. Censo 2004. Informe total país. Síntesis de resultados. 22 p. https://www.ine.gub.uy/documents/10181/3470 4/Informetotalpais.pdf/f5a3b680-2063-45ad9b69-7820e1ab7652 fecha de acceso 5 de febrero 2021.

Kareiva, P., y Marvier. M. (2012). What is Conservation Science? BioScience 62: 11.

Kellert, S. R. (2005). Building for life. Designing and understanding the human-nature connection. Washington: Island Press.

Kremen, C., Merenlender, A. M., y Murphy, D. D.
(1994). Ecological monitoring: a vital need for integrated conservation and development programs in tropics. Conservation Biology 8: 388-397.

Lindemann-Mathies, P. (2005). 'Loveable' mammals and 'lifeless' plants: How children's interest in common local organisms can be enhanced through observation of nature. International Journal of Science Education, 27 (6), 655-677.

Lindemann-Matthies, P., Constantinou, C., Lehnert, H.-J., Nagel, U., Raper, G., y Kadji-Beltran, C. (2011). Confidence and perceived competence of preservice teachers to implement biodiversity education in primary schools: Four comparative case studies from Europe. International Journal of Science Education, 33(16), 2247-2273.

Louv, R. (2010). Last child in the woods. London: Atlantic Books.

Mateos Jiménez, A., y García Fernández, B. (2016). Educar en la sostenibilidad durante la formación docente: posibilidades didácticas del Complejo Lagunar de Alcázar de San Juan [Educating in Sustainability during Teacher Training: Didactical Possibilities of the Wetland Complex of Alcázar de San Juan]. En: J. Rodríguez Torres (Coord.). Retos docentes universitarios como desafío curricular [University teaching challenges as a curricular challenge] pp. 429-444. Madrid: Mc Graw Hill.

Melero-Alcíbar, R., y Gamarra, P. (2016). Concepciones previas de futuros docentes sobre categorización animal: animales acuáticos. Revista electrónica de enseñanza de las ciencias, 15(2), 240-257.

Narvaez, M.C., Cueva, X.A., y Maldonado, M.E. (2019). Percepción humana: una herramienta para la conservación biológica. Museo de Investigaciones Zoológicas del IASA. Boletín Técnico 14, Serie Zoológica 14-15: 11-20.

Paraskevopoulos, S., Padeliadu, S., y Zafiropoulos, K. (1998). Environmental knowledge of elementary school students in Greece. The Journal of Environmental Education, 29(3), 5560.

Patrick., P., Byrne., J., Tunnicliffe., S., Asunta., T., Carvalho., G., Havu-Nuutinen., S., Sigurjónsdóttir., H., Óskarsdóttir., G., y Tracana., R. (2013). Students (ages 6, 10, and 15 years) in six countries knowledge of animals. NorDiNa, 9(1),18-32.

Patrick, P., y Tunnicliffle, S. (2011). What plants and animals do early childhood and primary students' name? Where do they see them? Journal of Science Education and Technology, 20(5), 630-642.

Pérez-Miles F. (1996). El conocimiento de la naturaleza en la cultura de los niños de Montevideo. Multiversidad, 6, 41-50.

Pergams, O., y Zaradic, P. (2006). Is love of nature in 
the US becoming love of electronic media? 16year downtrend in national park visits explained by watching movies, playing video games, internet use, and oil prices. Journal of Environmental Management, 80(4), 387-393.

Prokop, P., Kubiatko, M., y Fančovičová, J. (2008). Slovakian pupils' knowledge of, and attitudes toward, birds. Anthrozoös, 21(3), 221-235.

Prokop, P., y Rodák, R. (2009). Ability of Slovakian pupils to identify birds. Eurasia Journal of Mathematics, Science and Technology Education, 5(2), 127-133.

Randler, C. (2008). Pupils' factual knowlege about vertebrate species. Journal of Baltic Science Education, 7(1), 48-54.

Randler, C. (2010). Animal related activities as determinants of species knowledge. Eurasia Journal of Mathematics, Science \& Technology Education, 6 (4), 237-243.

Randler, C., Osti, J., y Hummel, E. (2012). Decline in interest in Biology among elementary school pupils during a generation. Eurasia Journal of Mathematics, Science and Technology Education, 8(3), 201-205.

Redford, K.H., y Robinson, J.G. (1991). Subsistence and commercial uses of wildlife in Latin America. En: J.G. Robinson, y K.H. Redford (Eds.). Neotropical wildlife use and conservation. The University of Chicago Press, Chicago, Illinois, USA.

Ripple, W.J., Wolf, C., Newsome, T.M., Galetti, M., Alamgir, M., Crist, E., Mahmoud, M.I., Laurance, W.F. y 15,364 scientist signatories from 184 countries. 2017. World scientists' warning to humanity: a second notice. BioScience, 67(12), 1026-1028.

Rodas, T.J., Estrada, A., Rau, J., y Morales, H.M. (2016). Uso local de los mamíferos no voladores entre los habitantes de Metzabok, El Tumbo y Laguna Colorada, Selva Lacandona, México. Revista Etnobiologia. 4(1): 39-50.

Salas, G. (2018). Un juego como estrategia de educación ambiental sobre la biodiversidad de Colombia. Tecné Episteme y Didaxis: TED, (44), 167-184.

Sánchez Emeterio, G., y García Fernández, B. (2013). Aprender para comunicar; bases de partida en entornos de humedales ibéricos [Learning to communicate; baselines in Iberian wetland environments]. Historia y Comunicación Social, 18, 777-788.

Scalfi, G.A.M. (2014). Fauna brasileira retratada na literatura infantil: instrumento para divulgação científica. Tesis de Maestría. UNICAMP. Campinas Brasil.

Scalfi, G., Barata, G., Salazar Granada, M., de Oliveira, M.M., Pascotto Garroti, y Furukawa Liberato, T. (2016). Animales notables: la percepción de los brasileños sobre la fauna en el billete del Real. Entreciencias: diálogos en la Sociedad del Conocimiento, 4 (11).

Seger, K.R., Solé, M., Martinez, R.A., y Schiavetti, A. (2019). Of people and toads: Local knowledge about amphibians around a protected area in the Brazilian Atlantic Forest. Neotropical Biology and Conservation, 14(4): 439-457.

Sepúlveda-Peña, J.C., Vejo-Paula, B., VillanuevaHedesa, C.A., González-Guerra, J.R., González-Alfonso, A., García-Lau, I., ... González-Moreno, M. (2019). Guatiní: un proyecto para fomentar el conocimiento de la avifauna cubana en las nuevas generaciones. Ciencia y Agricultura, 16(1), 17-30.

Serra Alanis, W.S., Scarabino, F., Wlodek, S., Furtado, G., y Balao, A. (2020). First record of 'Hemiancistrus' punctulatus Cardozo \& Malabarba, 1999 for Uruguay (SILURIFORMES: LORICARIIDAE). Boletín de la Sociedad Zoológica del Uruguay, 29 (1), 28-34.

Soga, M., y Gaston, K. (2016). Extinction of experience: The loss of human-nature interactions. Frontiers in Ecology and the Environment, 14(2), 94-101.

Soutullo, A., Clavijo, C., y Martínez-Lanfranco, J.A. (2013). Especies prioritarias para la conservación en Uruguay. Vertebrados, moluscos continentales y plantas vasculares. SNAP/DINAMA/MVOTMA y DICYT/MEC, Montevideo. $222 \mathrm{pp}$.

Torres, N., Salcedo, L., Becerra, A., y Valderrama, W. (2018). Fuentes de conocimiento en la identificación y preferencias de fauna en niñez de contextos rurales y urbanos. Revista Electrónica Educare, 22(3), 1-17.

Vaz-Canosa, P., y Rodríguez-Cajarville, M.J. (2015). Primer registro de Buteo brachyurus (Aves: Accipitriformes: Accipitridae) para Uruguay. 2015. Boletin de la Sociedad Zoologica del Uruguay (2a época), 24(1): 22-27.

Velarde, M.T. (2012). Importancia ecológica y cultural de una especie edémica de ajolote (Ambystoma dumerilii) del Lago de Pátzcuaro, Michoacán. Etnobiología, 10:40-49.

Vieira Santos, F.d.C., Medeiros Silva Souto W., Serejo Neves Ribeiro A., Farias Paiva de Lucena, R., y Guzzi, A. (2020). Traditional knowledge and perception of birds in the Parnaíba Delta environmental protection area, Northeast Brazil. Acta Scientiarum. Biological Sciences, 42 e47722.

Walsh, F. (2009). Human-Animal bonds I: The relation significance of companion animals. Family Process, 48(4), 462-480.

Wilson, E.O. (1984). Biophilia. The human bond with other species. Cambridge: Harvard University Press.

White, R., Eberstein, K., y Scott, D. (2018). Birds in the playground: Evaluating the effectiveness of an 
urban environmental education project in enhancing school children's awareness, knowledge and attitudes towards local wildlife. PLOS ONE, 13(3), 1-23.

Zapata-Ciro, J., Guevara, G., y Castaño-Villa, G.J. (2016). Conocimiento popular y perspectivas de conservación sobre las tortugas continentales en la parte baja del río la Miel (Colombia). Luna Azul, 43, 15-28.

Zuñiga, R.T. (1994). Abundancia relativa y uso tradicional del tepezcuintle (Agouti paca) en el Refugio de Vida Silvestre Barra del Colorado, Costa Rica. Tesis de Maestría. Programa Regional en Manejo de Vida Silvestre, Universidad Nacional de Costa Rica. Heredia, Costa Rica. 